International Journal of Pure and Applied Mathematics

Volume 90 No. $1 \quad 2014,85-100$

ISSN: 1311-8080 (printed version); ISSN: 1314-3395 (on-line version)

url: http://www.ijpam.eu

doi: http://dx.doi.org/10.12732/ijpam.v90i1.11

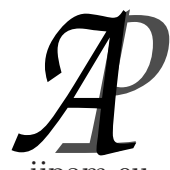

ijpam.eu

\title{
TWO FIXED POINT THEOREMS OF MAPPINGS SATISFYING CONTRACTIVE INEQUALITIES OF INTEGRAL TYPE
}

\author{
Zeqing $\mathrm{Liu}^{1}$, Bi Xu², Shin Min Kang ${ }^{3}$ \\ ${ }^{1,2}$ Department of Mathematics \\ Liaoning Normal University \\ Dalian, Liaoning, 116029, P.R. CHINA \\ ${ }^{3}$ Department of Mathematics and RINS \\ Gyeongsang National University \\ Jinju, 660-701, KOREA
}

\begin{abstract}
Two fixed point theorems of mappings satisfying contractive inequalities of integral type in complete metric spaces are proved. The results presented in this paper improve, unify and include a few results existing in literature.
\end{abstract}

Two illustrative examples are also provided.

AMS Subject Classification: $54 \mathrm{H} 25$

Key Words: fixed point, contractive inequality of integral type, complete metric space

\section{Introduction and Preliminaries}

In 2002, Branciari [2] extended the famous Banach's fixed point theorem by proving the following fixed point theorem for a contractive mapping of integral type.

Received: November 29, 2013

(c) 2014 Academic Publications, Ltd. url: www.acadpubl.eu 
Theorem 1.1. (see [2]) Let $f$ be a mapping from a complete metric space $(X, d)$ into itself satisfying

$$
\int_{0}^{d(f x, f y)} \varphi(t) d t \leq c \int_{0}^{d(x, y)} \varphi(t) d t, \quad \forall x, y \in X
$$

where $c \in(0,1)$ is a constant and $\varphi \in \Phi$. Then $f$ has a unique fixed point $a \in X$ such that $\lim _{n \rightarrow \infty} f^{n} x=a$ for each $x \in X$.

Afterwards, the authors in [1], [3]-[9] and others continued the study of the existence of fixed points and common fixed points for several contractive mappings of integral type in complete metric spaces, symmetric spaces and modular spaces. In 2011, Liu et al. [6] generalized the result of Branciari and established the following fixed point theorem.

Theorem 1.2. (see [6]) Let $f$ be a mapping from a complete metric space $(X, d)$ into itself satisfying

$$
\begin{aligned}
\int_{0}^{d(f x, f y)} \varphi(t) d t \leq \alpha(d(x, y)) & \int_{0}^{d(x, f x)} \varphi(t) d t \\
& +\beta(d(x, y)) \int_{0}^{d(y, f y)} \varphi(t) d t, \quad \forall x, y \in X,
\end{aligned}
$$

where $\varphi \in \Phi$ and $\alpha, \beta: \mathbb{R}^{+} \rightarrow[0,1)$ are two functions with $\alpha(t)+\beta(t)<1$ for all $t \in \mathbb{R}^{+}, \limsup _{s \rightarrow 0^{+}} \beta(s)<1$ and $\limsup _{s \rightarrow t^{+}} \frac{\alpha(s)}{1-\beta(s)}<1$ for all $t>0$.

Then $f$ has a unique fixed point $a \in X$ such that $\lim _{n \rightarrow \infty} f^{n} x=a$ for each $x \in X$.

The aim of this paper is to introduce two classes of mappings satisfying contractive inequalities of integral type as follows:

$$
\begin{gathered}
\int_{0}^{d(f x, f y)} \varphi(t) \leq \alpha(d(x, y)) \int_{0}^{d(x, f x)} \varphi(t) d t+\beta(d(x, y)) \int_{0}^{d(y, f y)} \varphi(t) d t \\
+\gamma(d(x, y)) \int_{0}^{d(x, y)} \varphi(t) d t, \quad \forall x, y \in X \\
\int_{0}^{2 d(f x, f y)} \varphi(t) d t \leq \alpha(d(x, y)) \int_{0}^{d(x, f y)} \varphi(t) d t+\beta(d(x, y)) \int_{0}^{d(y, f x)} \varphi(t) d t \\
+\gamma(d(x, y)) \int_{0}^{d(x, y)} \varphi(t) d t, \quad \forall x, y \in X
\end{gathered}
$$


and to study the existence of fixed points for the mappings (1.3) and (1.4). Under suitable conditions, we prove the existence of fixed points and convergence of iterative methods for the mappings (1.3) and (1.4) in complete metric spaces, which extend Theorems 1.1 and 1.2. Two nontrivial examples are included.

In the sequel, $\mathbb{R}^{+}=[0,+\infty), \mathbb{N}$ denotes the set of all positive integers, $\mathbb{N}_{0}=\{0\} \cup \mathbb{N}$, and

$\Phi=\left\{\varphi \mid \varphi: \mathbb{R}^{+} \rightarrow \mathbb{R}^{+}\right.$satisfies that $\varphi$ is Lebesgue integrable, summable on each compact subset of $\mathbb{R}^{+}$and $\int_{0}^{\varepsilon} \varphi(t) d t>0$ for each $\left.\varepsilon>0\right\}$.

Let $f$ be a mapping from a metric space $(X, d)$ into itself. For each $x \in X$ and $n \in \mathbb{N}_{0}$, put $d_{n}=d\left(f^{n} x, f^{n+1} x\right)$.

Lemma 1.3. (see [6]) Let $\varphi \in \Phi$ and $\left\{r_{n}\right\}_{n \in \mathbb{N}}$ be a nonnegative sequence with $\lim _{n \rightarrow \infty} r_{n}=a$. Then

$$
\lim _{n \rightarrow \infty} \int_{0}^{r_{n}} \varphi(t) d t=\int_{0}^{a} \varphi(t) d t .
$$

Lemma 1.4. (see [6]) Let $\varphi \in \Phi$ and $\left\{r_{n}\right\}_{n \in \mathbb{N}}$ be a nonnegative sequence. Then

$$
\lim _{n \rightarrow \infty} \int_{0}^{r_{n}} \varphi(t) d t=0 \Longleftrightarrow \lim _{n \rightarrow \infty} r_{n}=0 .
$$

\section{Main Results}

In this section, we show the existence and uniqueness of fixed points and iterative approximations for the mappings (1.3) and (1.4), respectively.

Theorem 2.1. Let $f$ be a mapping from a complete metric space $(X, d)$ into itself satisfying (1.3), $\varphi$ be in $\Phi$ and $\alpha, \beta, \gamma: \mathbb{R}^{+} \rightarrow[0,1)$ be functions with

$$
\begin{gathered}
\alpha(t)+\beta(t)+\gamma(t)<1, \quad \forall t \in \mathbb{R}^{+} \backslash\{0\} ; \\
\limsup _{s \rightarrow t}[\alpha(s)+\beta(s)+\gamma(s)]<1, \quad \forall t \in \mathbb{R}^{+} \backslash\{0\} ; \\
\min \left\{\limsup _{s \rightarrow 0^{+}} \alpha(s), \limsup _{s \rightarrow 0^{+}} \beta(s)\right\}<1 .
\end{gathered}
$$

Then $f$ has a unique fixed point $a \in X$ such that $\lim _{n \rightarrow \infty} f^{n} x=a$ for each $x \in X$. 
Proof. Let $x$ be an arbitrary point in $X$. If there exists some $n_{0} \in \mathbb{N}_{0}$ such that $d_{n_{0}}=0$, then $f^{n_{0}} x$ is a fixed point of $f$ and $f^{n_{0}} x=\lim _{n \rightarrow \infty} f^{n} x$. Now we assume that $d_{n}>0$ for each $n \in \mathbb{N}_{0}$. According to (1.3), we have

$$
\begin{aligned}
& \int_{0}^{d_{n}} \varphi(t) d t=\int_{0}^{d\left(f^{n} x, f^{n+1} x\right)} \varphi(t) d t \\
& \leq \alpha\left(d\left(f^{n-1} x, f^{n} x\right)\right) \int_{0}^{d\left(f^{n-1} x, f^{n} x\right)} \varphi(t) d t \\
& \quad+\beta\left(d\left(f^{n-1} x, f^{n} x\right)\right) \int_{0}^{d\left(f^{n} x, f^{n+1} x\right)} \varphi(t) d t \\
& \quad+\gamma\left(d\left(f^{n-1} x, f^{n} x\right)\right) \int_{0}^{d\left(f^{n-1} x, f^{n} x\right)} \varphi(t) d t \\
& =\alpha\left(d_{n-1}\right) \int_{0}^{d_{n-1}} \varphi(t) d t+\beta\left(d_{n-1}\right) \int_{0}^{d_{n}} \varphi(t) d t+\gamma\left(d_{n-1}\right) \int_{0}^{d_{n-1}} \varphi(t) d t \\
& =\left[\alpha\left(d_{n-1}\right)+\gamma\left(d_{n-1}\right)\right] \int_{0}^{d_{n-1}} \varphi(t) d t+\beta\left(d_{n-1}\right) \int_{0}^{d_{n}} \varphi(t) d t, \quad \forall n \in \mathbb{N} .
\end{aligned}
$$

Using (2.1) and (2.4), we obtain that

$$
\begin{aligned}
\int_{0}^{d_{n}} \varphi(t) d t & \leq \frac{\alpha\left(d_{n-1}\right)+\gamma\left(d_{n-1}\right)}{1-\beta\left(d_{n-1}\right)} \int_{0}^{d_{n-1}} \varphi(t) d t \\
& <\int_{0}^{d_{n-1}} \varphi(t) d t, \quad \forall n \in \mathbb{N}
\end{aligned}
$$

which yields that

$$
d_{n}<d_{n-1}, \quad \forall n \in \mathbb{N} .
$$

Note that (2.6) means that the sequence $\left\{d_{n}\right\}_{n \in \mathbb{N}}$ is decreasing. Consequently there exists a constant $c \geq 0$ with $\lim _{n \rightarrow \infty} d_{n}=c$.

Now we show that $c=0$. Otherwise $c>0$. In view of (2.4) and (2.6), we infer that

$$
\begin{aligned}
\int_{0}^{d_{n}} \varphi(t) d t & \leq\left[\alpha\left(d_{n-1}\right)+\gamma\left(d_{n-1}\right)\right] \int_{0}^{d_{n-1}} \varphi(t) d t+\beta\left(d_{n-1}\right) \int_{0}^{d_{n}} \varphi(t) d t \\
& \leq\left[\alpha\left(d_{n-1}\right)+\beta\left(d_{n-1}\right)+\gamma\left(d_{n-1}\right)\right] \int_{0}^{d_{n-1}} \varphi(t) d t, \quad \forall n \in \mathbb{N} .
\end{aligned}
$$

Taking upper limit in the above inequalities and using (2.2), $\varphi \in \Phi$ and Lemma 
1.1 , we conclude that

$$
\begin{aligned}
0 & <\int_{0}^{c} \varphi(t) d t=\limsup _{n \rightarrow \infty} \int_{0}^{d_{n}} \varphi(t) d t \\
& \leq \limsup _{n \rightarrow \infty}\left[\alpha\left(d_{n-1}\right)+\beta\left(d_{n-1}\right)+\gamma\left(d_{n-1}\right)\right] \limsup _{n \rightarrow \infty} \int_{0}^{d_{n-1}} \varphi(t) d t \\
& <\int_{0}^{c} \varphi(t) d t
\end{aligned}
$$

which is a contradiction. Hence

$$
\lim _{n \rightarrow \infty} d_{n}=0
$$

Next we prove that $\left\{f^{n} x\right\}_{n \in \mathbb{N}_{0}}$ is a Cauchy sequence. Suppose that $\left\{f^{n} x\right\}_{n \in \mathbb{N}_{0}}$ is not a Cauchy sequence, which means that there is a constant $\varepsilon>0$ such that for each positive integer $k$, there are positive integers $m(k)$ and $n(k)$ with $m(k)>n(k)>k$ such that

$$
d\left(f^{m(k)} x, f^{n(k)} x\right)>\varepsilon .
$$

For each positive integer $k$, let $m(k)$ denote the least integer exceeding $n(k)$ and satisfying (2.8). It follows that

$$
d\left(f^{m(k)} x, f^{n(k)} x\right)>\varepsilon \quad \text { and } \quad d\left(f^{m(k)-1} x, f^{n(k)} x\right) \leq \varepsilon, \quad \forall k \in \mathbb{N},
$$

which give that

$$
\begin{aligned}
\varepsilon & \leq d\left(f^{m(k)} x, f^{n(k)} x\right) \\
& \leq d\left(f^{m(k)-1} x, f^{m(k)} x\right)+d\left(f^{m(k)-1} x, f^{n(k)} x\right) \\
& \leq d_{m(k)-1}+\varepsilon, \quad \forall k \in \mathbb{N} .
\end{aligned}
$$

Letting $k \rightarrow \infty$ in the above inequalities and using (2.7), we get that

$$
\lim _{k \rightarrow \infty} d\left(f^{n(k)} x, f^{m(k)} x\right)=\varepsilon .
$$

It is easy to see that

$$
\begin{aligned}
& \left|d\left(f^{m(k)} x, f^{n(k)+1} x\right)-d\left(f^{m(k)} x, f^{n(k)} x\right)\right| \leq d_{n(k)}, \quad \forall k \in \mathbb{N} ; \\
& \left|d\left(f^{m(k)} x, f^{n(k)+1} x\right)-d\left(f^{m(k)+1} x, f^{n(k)+1} x\right)\right| \leq d_{m(k)}, \quad \forall k \in \mathbb{N} ; \\
& \left|d\left(f^{m(k)+1} x, f^{n(k)+2} x\right)-d\left(f^{m(k)+1} x, f^{n(k)+1} x\right)\right| \leq d_{n(k)+1}, \quad \forall k \in \mathbb{N} .
\end{aligned}
$$


Letting $k \rightarrow \infty$ in (2.10) and using (2.7) and (2.9), we conclude that

$$
\begin{aligned}
\varepsilon & =\lim _{k \rightarrow \infty} d\left(f^{m(k)} x, f^{n(k)+1} x\right)=\lim _{k \rightarrow \infty} d\left(f^{m(k)+1} x, f^{n(k)+1} x\right) \\
& =\lim _{k \rightarrow \infty} d\left(f^{m(k)+1} x, f^{n(k)+2} x\right) .
\end{aligned}
$$

By means of (1.3), (2.2), (2.7), (2.11), $\varphi \in \Phi$ and Lemmas 1.1 and 1.2, we have

$$
\begin{aligned}
& 0<\int_{0}^{\varepsilon} \varphi(t) d t=\limsup _{k \rightarrow \infty} \int_{0}^{d\left(f^{m(k)+1} x, f^{n(k)+2} x\right)} \varphi(t) d t \\
& \leq \limsup _{k \rightarrow \infty}\left[\alpha \left(d\left(f^{m(k)} x, f^{n(k)+1} x\right) \int_{0}^{d\left(f^{m(k)} x, f^{m(k)+1} x\right)} \varphi(t) d t\right.\right. \\
& +\beta\left(d\left(f^{m(k)} x, f^{n(k)+1} x\right)\right) \int_{0}^{d\left(f^{n(k)+1} x, f^{n(k)+2} x\right)} \varphi(t) d t \\
& \left.+\gamma\left(d\left(f^{m(k)} x, f^{n(k)+1} x\right)\right) \int_{0}^{d\left(f^{m(k)} x, f^{n(k)+1} x\right)} \varphi(t) d t\right] \\
& \leq \limsup _{k \rightarrow \infty}\left[\alpha \left(d\left(f^{m(k)} x, f^{n(k)+1} x\right)+\beta\left(d\left(f^{m(k)} x, f^{n(k)+1} x\right)\right)\right.\right. \\
& \left.+\gamma\left(d\left(f^{m(k)} x, f^{n(k)+1} x\right)\right)\right] \limsup _{k \rightarrow \infty} \max \left\{\int_{0}^{d_{m(k)}} \varphi(t) d t\right. \\
& \left.\quad \int_{0}^{d_{n(k)+1}} \varphi(t) d t, \int_{0}^{d\left(f^{m(k)} x, f^{n(k)+1} x\right)} \varphi(t) d t\right\} \\
& <\max \left\{0,0, \int_{0}^{\varepsilon} \varphi(t) d t\right\}=\int_{0}^{\varepsilon} \varphi(t) d t,
\end{aligned}
$$

which is impossible. Hence $\left\{f^{n} x\right\}_{n \in \mathbb{N}_{0}}$ is a Cauchy sequence. Since $(X, d)$ is a complete metric space, it follows that there exists a point $a \in X$ such that $\lim _{n \rightarrow \infty} f^{n} x=a$.

Now we assert that $a=f a$. Suppose that $a \neq f a$. We consider two possible case as follows:

Case 1. Assume that $\lim \sup _{s \rightarrow 0^{+}} \beta(s) \leq \limsup _{s \rightarrow 0^{+}} \alpha(s)$. In view of (1.3), 
(2.2), (2.3), (2.7), $\varphi \in \Phi$ and Lemmas 1.1 and 1.2, we arrive at

$$
\begin{aligned}
0< & \int_{0}^{d(a, f a)} \varphi(t) d t=\limsup _{n \rightarrow \infty} \int_{0}^{d\left(f^{n+1} x, f a\right)} \varphi(t) d t \\
\leq & \limsup _{n \rightarrow \infty}\left[\alpha\left(d\left(f^{n} x, a\right)\right) \int_{0}^{d\left(f^{n} x, f^{n+1} x\right)} \varphi(t) d t\right. \\
& \left.\quad+\beta\left(d\left(f^{n} x, a\right)\right) \int_{0}^{d(a, f a)} \varphi(t) d t+\gamma\left(d\left(f^{n} x, a\right)\right) \int_{0}^{d\left(f^{n} x, a\right)} \varphi(t) d t\right] \\
\leq & \limsup _{n \rightarrow \infty} \alpha\left(d\left(f^{n} x, a\right)\right) \limsup _{n \rightarrow \infty} \int_{0}^{d_{n}} \varphi(t) d t \\
& +\limsup _{n \rightarrow \infty} \beta\left(d\left(f^{n} x, a\right)\right) \limsup _{n \rightarrow \infty} \int_{0}^{d(a, f a)} \varphi(t) d t \\
& +\limsup _{n \rightarrow \infty} \gamma\left(d\left(f^{n} x, a\right)\right) \limsup _{n \rightarrow \infty} \int_{0}^{d\left(f^{n} x, a\right)} \varphi(t) d t \\
< & \int_{0}^{d(a, f a)} \varphi(t) d t,
\end{aligned}
$$

which is a contradiction;

Case 2. Assume that $\limsup _{s \rightarrow 0^{+}} \alpha(s)<\limsup _{s \rightarrow 0^{+}} \beta(s)$. By virtue of (1.3), (2.2), (2.3), (2.7), $\varphi \in \Phi$ and Lemmas 1.1 and 1.2, we get that

$$
\begin{aligned}
0< & \int_{0}^{d(f a, a)} \varphi(t) d t=\limsup _{n \rightarrow \infty} \int_{0}^{d\left(f a, f^{n+1} x\right)} \varphi(t) d t \\
\leq & \limsup _{n \rightarrow \infty}\left[\alpha\left(d\left(a, f^{n} x\right)\right) \int_{0}^{d(a, f a)} \varphi(t) d t\right. \\
& \left.\quad+\beta\left(d\left(a, f^{n} x\right)\right) \int_{0}^{d\left(f^{n} x, f^{n+1} x\right)} \varphi(t) d t+\gamma\left(d\left(a, f^{n} x\right)\right) \int_{0}^{d\left(a, f^{n} x\right)} \varphi(t) d t\right] \\
\leq & \limsup _{n \rightarrow \infty} \alpha\left(d\left(a, f^{n} x\right)\right) \limsup _{n \rightarrow \infty} \int_{0}^{d(f a, a)} \varphi(t) d t \\
& +\limsup _{n \rightarrow \infty} \beta\left(d\left(a, f^{n} x\right)\right) \limsup _{n \rightarrow \infty} \int_{0}^{d_{n}} \varphi(t) d t \\
& +\limsup _{n \rightarrow \infty} \gamma\left(d\left(a, f^{n} x\right)\right) \limsup _{n \rightarrow \infty} \int_{0}^{d\left(a, f^{n} x\right)} \varphi(t) d t \\
< & \int_{0}^{d(f a, a)} \varphi(t) d t,
\end{aligned}
$$

which is absurd. In conclusion, we infer that $a=f a$. 
In the end, we prove that $a$ is the unique fixed point of $f$ in $X$. Suppose that $f$ has another fixed point $b \in X \backslash\{a\}$. It follows from $\varphi \in \Phi,(1.3)$ and (2.1) that

$$
\begin{aligned}
0< & \int_{0}^{d(a, b)} \varphi(t) d t=\int_{0}^{d(f a, f b)} \varphi(t) d t \\
\leq & \alpha(d(a, b)) \int_{0}^{d(a, f a)} \varphi(t) d t+\beta(d(a, b)) \int_{0}^{d(b, f b)} \varphi(t) d t \\
& +\gamma(d(a, b)) \int_{0}^{d(a, b)} \varphi(t) d t \\
= & \gamma(d(a, b)) \int_{0}^{d(a, b)} \varphi(t) d t<\int_{0}^{d(a, b)} \varphi(t) d t
\end{aligned}
$$

which is impossible. This completes the proof.

Remark 2.2. If $\alpha(t)=\beta(t)=0$ and $\gamma(t)=c$ for all $t \in \mathbb{R}^{+}$, then Theorem 2.1 develops into Theorem 1.1; if $\gamma(t)=0$ for all $t \in \mathbb{R}^{+}$, then Theorem 2.1 reduces to Theorem 1.2. The following example explains that Theorem 2.1 extends Theorem 1.1.

Example 2.3. Let $X=\mathbb{R}$ be endowed with the Euclidean metric $d=|\cdot|$, $f: X \rightarrow X, \varphi: \mathbb{R}^{+} \rightarrow \mathbb{R}^{+}$and $\alpha, \beta, \gamma: \mathbb{R}^{+} \rightarrow[0,1)$ be defined by

$$
\begin{gathered}
f(x)=\frac{|x|}{1+|x|}, \quad \forall x \in X, \\
\varphi(t)=3 t^{2}, \quad \alpha(t)=\frac{2 t}{(1+t)^{3}}, \quad \beta(t)=\frac{t^{3}}{(1+t)^{3}}, \quad \forall t \in \mathbb{R}^{+}
\end{gathered}
$$

and

$$
\gamma(t)= \begin{cases}\frac{1+t^{2}}{(1+t)^{3}}, & \forall t>0 \\ \frac{1}{4}, & t=0\end{cases}
$$


Clearly, $\varphi \in \Phi$ and (2.2)-(2.3) hold. Note that

$$
\begin{aligned}
\int_{0}^{d(f x, f y)} \varphi(t) d t= & \left|\frac{|x|}{1+|x|}-\frac{|y|}{1+|y|}\right|^{3}=\frac{|| x|-| y||^{3}}{(1+|x|)^{3}(1+|y|)^{3}} \\
\leq & \frac{|x-y|^{3}}{(1+|x|)^{3}(1+|y|)^{3}} \leq \frac{1+|x-y|^{2}}{(1+|x-y|)^{3}}|x-y|^{3} \\
\leq & \gamma(d(x, y)) \int_{0}^{d(x, y)} \varphi(t) d t \\
\leq & \alpha(d(x, y)) \int_{0}^{d(x, f x)} \varphi(t) d t+\beta(d(x, y)) \int_{0}^{d(y, f y)} \varphi(t) d t \\
& +\gamma(d(x, y)) \int_{0}^{d(x, y)} \varphi(t) d t, \quad \forall x, y \in X,
\end{aligned}
$$

which yields (1.3). Hence the conditions of Theorem 2.1 are fulfilled. It follows from Theorem 2.1 that $f$ has a unique fixed point $0 \in X$. But we can not invoke Theorem 1.1 to show the existence of a fixed point of $f$ in $X$ because (1.1) does not hold. Suppose that (1.1) holds. It follows that

$$
\begin{aligned}
\int_{0}^{d(f x, f y)} \varphi(t) d t & =\frac{|| x|-| y||^{3}}{(1+|x|)^{3}(1+|y|)^{3}} \\
& \leq c|x-y|^{3}=c \int_{0}^{d(x, y)} \varphi(t) d t, \quad \forall x, y \in X,
\end{aligned}
$$

which gives that

$$
\frac{1}{(1+|x|)^{3}(1+|y|)^{3}} \leq c, \quad \forall x, y \in \mathbb{R}^{+}-\{0\} \text { with } x \neq y,
$$

which means that

$$
1=\lim _{\substack{(x, y) \rightarrow(0,0) \\ x \neq y}} \frac{1}{(x+1)^{3}(y+1)^{3}} \leq c<1,
$$

which is impossible.

Theorem 2.4. Let $f$ be a mapping from a complete metric space $(X, d)$ into itself satisfying (1.4), $\varphi$ be in $\Phi$ and $\alpha, \beta, \gamma: \mathbb{R}^{+} \rightarrow[0,1)$ be functions with (2.1)-(2.3). Then $f$ has a unique fixed point $a \in X$ such that $\lim _{n \rightarrow \infty} f^{n} x=a$ for each $x \in X$. 
Proof. Let $x$ be an arbitrary point in $X$. Without loss of generality, we assume that $d_{n}>0$ for each $n \in \mathbb{N}_{0}$. Suppose that (2.6) does not hold. That is, there exists some $n_{0} \in \mathbb{N}$ satisfying

$$
d_{n_{0}} \geq d_{n_{0}-1}
$$

On account of (1.4), (2.1), (2.12) and $\varphi \in \Phi$, we conclude immediately that

$$
\begin{aligned}
0< & \int_{0}^{2 d_{n_{0}}} \varphi(t) d t=\int_{0}^{2 d\left(f^{n_{0}} x, f^{n_{0}+1} x\right)} \varphi(t) d t \\
\leq & \alpha\left(d\left(f^{n_{0}-1} x, f^{n_{0}} x\right)\right) \int_{0}^{d\left(f^{n_{0}-1} x, f^{n_{0}+1} x\right)} \varphi(t) d t \\
& +\beta\left(d\left(f^{n_{0}-1} x, f^{n_{0}} x\right)\right) \int_{0}^{d\left(f^{n_{0}} x, f^{n_{0}} x\right)} \varphi(t) d t \\
& +\gamma\left(d\left(f^{n_{0}-1} x, f^{n_{0}} x\right)\right) \int_{0}^{d\left(f^{n_{0}-1} x, f^{n_{0}} x\right)} \varphi(t) d t \\
\leq & \alpha\left(d_{n_{0}-1}\right) \int_{0}^{d_{n_{0}-1}+d_{n_{0}}} \varphi(t) d t+\gamma\left(d_{n_{0}-1}\right) \int_{0}^{d_{n_{0}-1}} \varphi(t) d t \\
\leq & {\left[\alpha\left(d_{n_{0}-1}\right)+\gamma\left(d_{n_{0}-1}\right)\right] \int_{0}^{2 d_{n_{0}}} \varphi(t) d t } \\
< & \int_{0}^{2 d_{n_{0}}} \varphi(t) d t
\end{aligned}
$$

which is a contradiction. Hence (2.6) holds. Consequently, there exists a constant $c \geq 0$ with $\lim _{n \rightarrow \infty} d_{n}=c$. Suppose that $c>0$. Making use of (1.4), (2.2), (2.6), $\varphi \in \Phi$ and Lemma 1.1, we have

$$
\begin{aligned}
0< & \int_{0}^{2 c} \varphi(t) d t=\limsup _{n \rightarrow \infty} \int_{0}^{2 d_{n}} \varphi(t) d t \\
= & \limsup _{n \rightarrow \infty} \int_{0}^{2 d\left(f^{n} x, f^{n+1} x\right)} \varphi(t) d t \\
\leq & \limsup _{n \rightarrow \infty}\left[\alpha\left(d\left(f^{n-1} x, f^{n} x\right)\right) \int_{0}^{d\left(f^{n-1} x, f^{n+1} x\right)} \varphi(t) d t\right. \\
& +\beta\left(d\left(f^{n-1} x, f^{n} x\right)\right) \int_{0}^{d\left(f^{n} x, f^{n} x\right)} \varphi(t) d t \\
& \left.+\gamma\left(d\left(f^{n-1} x, f^{n} x\right)\right) \int_{0}^{d\left(f^{n-1} x, f^{n} x\right)} \varphi(t) d t\right]
\end{aligned}
$$




$$
\begin{aligned}
& \leq \limsup _{n \rightarrow \infty}\left[\alpha\left(d_{n-1}\right) \int_{0}^{d_{n-1}+d_{n}} \varphi(t) d t+\gamma\left(d_{n-1}\right) \int_{0}^{d_{n-1}} \varphi(t) d t\right] \\
& \leq \limsup _{n \rightarrow \infty}\left[\alpha\left(d_{n-1}\right)+\gamma\left(d_{n-1}\right)\right] \limsup _{n \rightarrow \infty} \int_{0}^{2 d_{n-1}} \varphi(t) d t \\
& <\int_{0}^{2 c} \varphi(t) d t,
\end{aligned}
$$

which is impossible. Hence $c=0$ and (2.7) holds. Suppose that $\left\{f^{n} x\right\}_{n \in \mathbb{N}}$ is not a Cauchy sequence, which means that there is a constant $\varepsilon>0$ such that for each positive integer $k$, there are positive integers $m(k)$ and $n(k)$ with $m(k)>n(k)>k$ such that

$$
d\left(f^{m(k)} x, f^{n(k)} x\right)>\varepsilon .
$$

For each positive integer $k$, let $m(k)$ denote the least integer exceeding $n(k)$ and satisfying the above inequality. As in the proof of Theorem 2.1, we infer similarly that (2.9)-(2.11) hold. Note that (2.7) ensures that

$$
\left|d\left(f^{m(k)} x, f^{n(k)+2} x\right)-d\left(f^{m(k)+1} x, f^{n(k)+2} x\right)\right| \leq d_{m(k)} \rightarrow 0 \quad \text { as } k \rightarrow \infty,
$$

which together with (2.11) yields that

$$
\lim _{k \rightarrow \infty} d\left(f^{m(k)} x, f^{n(k)+2} x\right)=\varepsilon .
$$

In light of (1.4), (2.2), (2.9), (2.11), (2.13), $\varphi \in \Phi$ and Lemma 1.1, we know that

$$
\begin{aligned}
0< & \int_{0}^{2 \varepsilon} \varphi(t) d t=\limsup _{k \rightarrow \infty} \int_{0}^{2 d\left(f^{m(k)+1} x, f^{n(k)+2} x\right)} \varphi(t) d t \\
\leq & \limsup _{k \rightarrow \infty}\left[\alpha\left(d\left(f^{m(k)} x, f^{n(k)+1} x\right)\right) \int_{0}^{d\left(f^{m(k)} x, f^{n(k)+2} x\right)} \varphi(t) d t\right. \\
& +\beta\left(d\left(f^{m(k)} x, f^{n(k)+1} x\right)\right) \int_{0}^{d\left(f^{n(k)+1} x, f^{m(k)+1} x\right)} \varphi(t) d t \\
& \left.+\gamma\left(d\left(f^{m(k)} x, f^{n(k)+1} x\right)\right) \int_{0}^{d\left(f^{m(k)} x, f^{n(k)+1} x\right)} \varphi(t) d t\right] \\
\leq & \limsup _{k \rightarrow \infty}\left[\alpha\left(d\left(f^{m(k)} x, f^{n(k)+1} x\right)\right)+\beta\left(d\left(f^{m(k)} x, f^{n(k)+1} x\right)\right)\right. \\
& \left.+\gamma\left(d\left(f^{m(k)} x, f^{n(k)+1} x\right)\right)\right] \limsup _{k \rightarrow \infty} \max \left\{\int_{0}^{d\left(f^{m(k)} x, f^{n(k)+2} x\right)} \varphi(t) d t,\right.
\end{aligned}
$$




$$
\begin{aligned}
& \left.\int_{0}^{d\left(f^{n(k)+1} x, f^{m(k)+1} x\right)} \varphi(t) d t, \int_{0}^{d\left(f^{m(k)} x, f^{n(k)+1} x\right)} \varphi(t) d t\right\} \\
< & \int_{0}^{\varepsilon} \varphi(t) d t \leq \int_{0}^{2 \varepsilon} \varphi(t) d t,
\end{aligned}
$$

which is impossible. Thus $\left\{f^{n} x\right\}_{n \in \mathbb{N}}$ is a Cauchy sequence. Since $(X, d)$ is a complete metric space, it follows that there exists a point $a \in X$ such that $\lim _{n \rightarrow \infty} f^{n} x=a$. Suppose that $a \neq f a$. We consider two possible cases as follows:

Case 1. Let $\limsup _{s \rightarrow 0^{+}} \alpha(s) \geq \limsup _{s \rightarrow 0^{+}} \beta(s)$. In view of (1.4), (2.3), $\varphi \in \Phi$ and Lemmas 1.1 and 1.2, we conclude that

$$
\begin{aligned}
& 0<\int_{0}^{2 d(f a, a)} \varphi(t) d t=\limsup _{n \rightarrow \infty} \int_{0}^{2 d\left(f a, f^{n+1} x\right)} \varphi(t) d t \\
& \leq \limsup _{n \rightarrow \infty}\left[\alpha\left(d\left(a, f^{n} x\right)\right) \int_{0}^{d\left(a, f^{n+1} x\right)} \varphi(t) d t\right. \\
& \left.\quad+\beta\left(d\left(a, f^{n} x\right)\right) \int_{0}^{d\left(f^{n} x, f a\right)} \varphi(t) d t+\gamma\left(d\left(a, f^{n} x\right)\right) \int_{0}^{d\left(a, f^{n} x\right)} \varphi(t) d t\right] \\
& \leq \limsup _{n \rightarrow \infty}\left[\alpha\left(d\left(a, f^{n} x\right)\right)+\gamma\left(d\left(a, f^{n} x\right)\right)\right] \\
& \quad \times \limsup _{n \rightarrow \infty} \max \left\{\int_{0}^{d\left(a, f^{n+1} x\right)} \varphi(t) d t, \int_{0}^{d\left(a, f^{n} x\right)} \varphi(t) d t\right\} \\
& \quad+\limsup _{n \rightarrow \infty} \beta\left(d\left(a, f^{n} x\right)\right) \limsup _{n \rightarrow \infty} \int_{0}^{d\left(f a, f^{n} x\right)} \varphi(t) d t \\
& <\int_{0}^{d(f a, a)} \varphi(t) d t \leq \int_{0}^{2 d(f a, a)} \varphi(t) d t,
\end{aligned}
$$

which is irreconcilable;

Case 2. Let $\limsup _{s \rightarrow 0^{+}} \alpha(s)<\limsup _{s \rightarrow 0^{+}} \beta(s)$. By virtue of (1.4), (2.3), $\varphi \in \Phi$ and Lemmas 1.1 and 1.2, we get that

$$
\begin{aligned}
& 0<\int_{0}^{2 d(a, f a)} \varphi(t) d t=\limsup _{n \rightarrow \infty} \int_{0}^{2 d\left(f^{n+1} x, f a\right)} \varphi(t) d t \\
& \leq \limsup _{n \rightarrow \infty}\left[\alpha\left(d\left(f^{n} x, a\right)\right) \int_{0}^{d\left(f^{n} x, f a\right)} \varphi(t) d t\right. \\
& \left.\quad+\beta\left(d\left(f^{n} x, a\right)\right) \int_{0}^{d\left(a, f^{n+1} x\right)} \varphi(t) d t+\gamma\left(d\left(f^{n} x, a\right)\right) \int_{0}^{d\left(f^{n} x, a\right)} \varphi(t) d t\right]
\end{aligned}
$$




$$
\begin{aligned}
& \leq \limsup _{n \rightarrow \infty} \alpha\left(d\left(f^{n} x, a\right)\right) \cdot \limsup _{n \rightarrow \infty} \int_{0}^{d\left(f^{n} x, f a\right)} \varphi(t) d t \\
& +\limsup _{n \rightarrow \infty}\left[\beta\left(d\left(f^{n} x, a\right)\right)+\gamma\left(d\left(f^{n} x, a\right)\right)\right] \\
& \quad \times \limsup _{n \rightarrow \infty} \max \left\{\int_{0}^{d\left(a, f^{n+1} x\right)} \varphi(t) d t, \int_{0}^{d\left(f^{n} x, a\right)} \varphi(t) d t\right\} \\
& <\int_{0}^{d(a, f a)} \varphi(t) d t \leq \int_{0}^{2 d(a, f a)} \varphi(t) d t,
\end{aligned}
$$

which is impossible. Hence $a=f a$, that is, $a$ is a fixed point of $f$ in $X$. Suppose that $f$ has another fixed point $b \in X \backslash\{a\}$. It follows from (1.4), (2.2) and $\varphi \in \Phi$ that

$$
\begin{aligned}
0< & \int_{0}^{2 d(a, b)} \varphi(t) d t=\int_{0}^{2 d(f a, f b)} \varphi(t) d t \\
\leq & \alpha(d(a, b)) \int_{0}^{d(a, f b)} \varphi(t) d t+\beta(d(a, b)) \int_{0}^{d(b, f a)} \varphi(t) d t \\
& +\gamma(d(a, b)) \int_{0}^{d(a, b)} \varphi(t) d t \\
= & {[\alpha(d(a, b))+\beta(d(a, b))+\gamma(d(a, b))] \int_{0}^{d(a, b)} \varphi(t) d t } \\
< & \int_{0}^{d(a, b)} \varphi(t) d t \leq \int_{0}^{2 d(a, b)} \varphi(t) d t,
\end{aligned}
$$

which is paradoxical. This completes the proof.

Remark 2.5. The following example is an application of Theorem 2.4.

Example 2.6. Let $X=\mathbb{R}^{+}$be endowed with the Euclidean metric $d=|\cdot|$, $f: X \rightarrow X, \varphi: \mathbb{R}^{+} \rightarrow \mathbb{R}^{+}$and $\alpha, \beta, \gamma: \mathbb{R}^{+} \rightarrow[0,1)$ be defined by

$$
\begin{gathered}
f(x)= \begin{cases}0, & \forall x \in\left[0,2^{\frac{1}{2}}\right), \\
\frac{1}{2 x}, & \forall x \in\left[2^{\frac{1}{2}},+\infty\right),\end{cases} \\
\varphi(t)=2 t, \quad \forall t \in \mathbb{R}^{+}, \quad \alpha(t)=\beta(t)=\gamma(t)= \begin{cases}\frac{209}{630}, & t=0, \\
\frac{t+1}{4 t+3}, & \forall t \in(0,+\infty) .\end{cases}
\end{gathered}
$$

Clearly, (2.1)-(2.3) hold. In order to verify (1.4), we have to consider the following three cases for any $x, y \in X$ with $y<x$ : 
Case 1. Let $x, y \in X$ with $0 \leq y<x<2^{\frac{1}{2}}$. It is clear that

$$
\begin{aligned}
\int_{0}^{2 d(f x, f y)} \varphi(t) d t & =0 \\
\leq & \alpha(d(x, y)) \int_{0}^{d(x, f y)} \varphi(t) d t+\beta(d(x, y)) \int_{0}^{d(y, f x)} \varphi(t) d t \\
& +\gamma(d(x, y)) \int_{0}^{d(x, y)} \varphi(t) d t
\end{aligned}
$$

Case 2. Let $x, y \in X$ with $0 \leq y<2^{\frac{1}{2}} \leq x$. It follows that

$$
\begin{aligned}
\int_{0}^{2 d(f x, f y)} \varphi(t) d t & =\frac{1}{x^{2}} \leq \frac{1}{2}=\frac{1}{4} \times 2 \\
\leq & \frac{1}{4} x^{2}<\left[\frac{x-y+1}{4(x-y)+3}\right] x^{2} \\
\leq & \alpha(d(x, y)) \int_{0}^{d(x, f y)} \varphi(t) d t \\
\leq & \alpha(d(x, y)) \int_{0}^{d(x, f y)} \varphi(t) d t+\beta(d(x, y)) \int_{0}^{d(y, f x)} \varphi(t) d t \\
& +\gamma(d(x, y)) \int_{0}^{d(x, y)} \varphi(t) d t
\end{aligned}
$$

Case 3. Let $x, y \in X$ with $2^{\frac{1}{2}} \leq y<x$. It is easy to show that

$$
\begin{aligned}
\int_{0}^{2 d(f x, f y)} \varphi(t) d t= & \left(\frac{1}{x}-\frac{1}{y}\right)^{2}=\frac{(x-y)^{2}}{(x y)^{2}}<\frac{1}{4}(x-y)^{2} \\
< & {\left[\frac{x-y+1}{4(x-y)+3}\right](x-y)^{2} } \\
\leq & \gamma(d(x, y))(x-y)^{2} \\
\leq & \alpha(d(x, y)) \int_{0}^{d(x, f y)} \varphi(t) d t+\beta(d(x, y)) \int_{0}^{d(y, f x)} \varphi(t) d t \\
& +\gamma(d(x, y)) \int_{0}^{d(x, y)} \varphi(t) d t
\end{aligned}
$$

Hence (1.4) holds. Thus Theorem 2.4 ensures that $f$ has a unique fixed point $0 \in X$ and $\lim _{n \rightarrow \infty} f^{n} x=0$ for each $x \in X$. 


\section{Acknowledgments}

This research was supported by the Science Research Foundation of Educational Department of Liaoning Province (L2012380).

\section{References}

[1] I. Altun, D. Türkoğlu, B.E. Rhoades, Fixed points of weakly compatible maps satisfying a general contractive condition of integral type, Fixed Point Theory Appl., 2007 (2007), Article ID 17301, 9 pages, doi: $10.1155 / 2007 / 17301$

[2] A. Branciari, A fixed point theorem for mappings satisfying a general contractive condition of integral type, Int. J. Math. Math. Sci., 29, No. 9 (2002), 531-536, doi: 10.1155/S0161171202007524

[3] A. Djoudi, A. Aliouche, Common fixed point theorems of Gregus type for weakly compatible mappings satisfying contractive conditions of integral type, J. Math. Anal. Appl., 329, No. 1 (2007), 31-45, doi: 10.1016/j.jmaa.2006.06.037

[4] A. Djoudi, F. Merghadi, Common fixed point theorems for maps under a contractive condition of integral type, J. Math. Anal. Appl., 341, No. 2 (2008), 953-960, doi: 10.1016/j.jmaa.2007.10.064

[5] J. Jachymski, Remarks on contractive conditions of integral type, Nonlinear Anal., 71, No. 3-4 (2009), 1073-1081, doi: 10.1016/j.na.2008.11.046

[6] Z. Liu, X. Li, S.M. Kang, S.Y. Cho, Fixed point theorems for mappings satisfying contractive conditions of integral type and applications, Fixed Point Theory Appl., 2011, No. 64 (2011), 18 pages, doi: 10.1186/16871812-2011-64

[7] B.E. Rhoades, Two fixed point theorems for mappings satisfying a general contractive condition of integral type, Int. J. Math. Math. Sci., 2003, No. 63 (2003), 4007-4013, doi: 10.1155/S0161171203208024

[8] T. Suzuki, Meir-Keeler contractions of integral type are still Meir-Keeler contractions, Int. J. Math. Math. Sci., 2007 (2007), Article ID 39281, 6 pages, doi: $10.1155 / 2007 / 39281$ 
[9] P. Vijayaraju, B.E. Rhoades, R. Mohanraj, A fixed point theorem for a pair of maps satisfying a general contractive condition of integral type, Int. J. Math. Math. Sci., 2005, No. 15 (2005), 2359-2364, doi: 10.1155/IJMMS.2005.2359 